\title{
Efficacy of Edaravone in Cardioembolic Stroke
}

\author{
Yuichiro Inatomi ${ }^{1}$, Tomohiro Takita ${ }^{1}$, Toshiro Yonehara ${ }^{1}$, Shodo Fujioka ${ }^{1}$, \\ Yoichiro Hashimoto ${ }^{2}$, Teruyuki Hirano ${ }^{3}$ and Makoto Uchino ${ }^{3}$
}

\begin{abstract}
Objective We investigated the efficacy of edaravone in patients with cardioembolic stroke.

Methods Cardioembolic stroke patients were treated with drip intravenous infusion of edaravone (ED group, $\mathrm{n}=141$ ) for 7 days, and were retrospectively compared with a historical-controlled cohort of similar patients (control group, $\mathrm{n}=114$ ).

Results Early improvement (between day 0 and day 10), defined as change in National Institutes of Health Stroke Scale (NIHSS), was seen more frequently in mild patients (NIHSS on admission $\leq 7$ ) among the ED group than in the control group (change in NIHSS +2 vs. -2 , respectively, $p=0.013$ ). Similar efficacy was not seen in the moderate to severe (NIHSS >7) patients. Independent patients (modified Rankin Scale $\leq 2$ ) 6 months after the onset were likely to be less frequent in the ED than the control group (28\% versus $41 \%$; $p=$ 0.066). Other clinical outcomes in the ED group were not significantly different from those in the control group.

Conclusion The results suggest that edaravone may only be effective in mild patients with cardioembolic stroke.
\end{abstract}

Key words: edaravone, brain infarction, neuroprotective agent, ischemic penumbra, cardioembolic stroke

(DOI: 10.2169/internalmedicine.45.1423)

\section{Introduction}

The efficacy of edaravone, a free radical scavenger, has been verified in acute ischemic stroke in a double-blind, randomized, placebo-controlled study (1), and has been used in clinical practice in Japan. In a rat brain ischemiareperfusion model, edaravone has been shown to ameliorate brain edema $(2,3)$ and tissue injury (4-6), and to delay neuronal death (7) and neurological deficits $(3,5)$. On the basis of such pharmacological effects, it is suggested that edaravone may be more effective for treating patients with cardioembolic stroke than for those with other subtypes of ischemic stroke. Patients with cardioembolic stroke may experience successful thrombolysis and natural reperfusion, resulting in a so-called spectacular shrinking deficit (8).

To our knowledge, randomized controlled studies of the efficacy of edaravone in treating acute ischemic stroke have not been reported except for the study conducted by the Edaravone Acute Brain Infarction Study Group (1). In this report the outcome parameters were evaluated for the entire group of patients, but not in patients stratified according to clinical subtypes or severity. Furthermore, the endpoints in this study were only long-term activity of daily life (ADL) but not improvement in acute phase indices, which might indicate the presence of a neuroprotective effect at an early stage of reperfusion. There were two other non-randomized controlled studies previously reported $(9,10)$, however the patients in these studies were heterogeneous, including internal carotid artery occlusion (9), and atherothrombotic and lacunar strokes (10). Case-controlled studies analyzing the efficacy of edaravone in cardioembolic stroke have not been reported to date.

The present retrospective historical-controlled study was conducted to investigate the efficacy of edaravone in patients with acute ischemic stroke. In this study, the subjects were limited to patients with cardioembolic stroke, improvement in acute phase as well as long-term ADL was evaluated, and outcome parameters were analyzed by stratification of clinical severity.

\footnotetext{
${ }^{1}$ Stroke Center, Saiseikai Kumamoto Hospital, Kumamoto, ${ }^{2}$ Department of Neurology, Kumamoto City Hospital, Kumamoto and ${ }^{3}$ Department of Neurology, Graduate School of Medical Sciences, Kumamoto University, Kumamoto

Received for publication May 18, 2005; Accepted for publication December 10, 2005

Correspondence to Yuichiro Inatomi, Stroke Center, Saiseikai Kumamoto Hospital, 5-3-1 Chikami, Kumamoto 861-4193
} 


\section{Methods}

\section{Background of the study}

This was a historical-controlled trial in consecutive patients with acute ischemic stroke, who were admitted to our hospital from October 1, 2000 to September 30, 2002. Eligible patients were admitted to our hospital within 7 days after the onset of brain infarction. They were initially evaluated by neurologists using the National Institutes of Health Stroke Scale (NIHSS) (11) on admission (day 0). Laboratory examinations, brain CT scan, chest X-rays, 12-lead electrocardiograms, carotid echography, transthoracic echocardiography, and 24-hour ECG monitoring were performed in all patients. Magnetic resonance imaging and transesophageal echocardiography were also performed when indicated, and with the permission of patients.

Patients with a tentative diagnosis of cardioembolic stroke on admission were treated with intravenous or selective intra-arterial thrombolysis when our inclusion criteria $(\leq 80$ years old; admission within 3 hours after onset of symptoms; evidence of occlusion of the middle cerebral artery) were satisfied. They were treated with intravenous glycerol and in some instances with aspirin and/or warfarin from day 2. On the other hand, patients with a tentative diagnosis of small vessel or atherosclerotic stroke were treated with antithrombotic agents including ozagrel sodium, argatroban, and aspirin.

Edaravone has been clinically available since June 1, 2001 in Japan. Criteria for administration of edaravone in our study included: 1) tentative diagnosis of cardioembolic stroke; 2) initiation of treatment within 24 hours of onset of symptoms; 3) serum creatinine $<2.0 \mathrm{mg} / \mathrm{dl}$. In our institute the final decision whether to administer the agent was made by each attending physician. Thirty $\mathrm{mg}$ of edaravone diluted with $100 \mathrm{ml}$ of saline was administered by intravenous drip infusion over a period of 30 minutes, every 12 hours for 7 days. The criteria adopted for patient discharge or transfer to rehabilitation institutes were 1) withdrawal of treatment in the acute phase, and 2) no active complications.

\section{Patients}

Subjects were admitted to our hospital within 24 hours after the onset of symptoms during the 24-month study period. Diagnosis of cardioembolic stroke was made according to the definitions given in the Trial of ORG 10172 in Acute Stroke Treatment (TOAST) study (12).

These patients were divided into three groups: 1) a control group of patients admitted before June 1, 2001, who were not treated with edaravone; 2) a treatment group (ED group) of patients admitted after that day, who were treated with edaravone; 3) a patient group (excluded group) also admitted subsequently but not treated with edaravone in which the attending physician initially did not make a tentative diagnosis of cardioembolic stroke. Among these three groups, the control group and ED group were designated as subject groups in which outcome parameters were statistically compared.

\section{Clinical characteristics}

The following risk factors were assessed: 1) presence of hypertension, defined as the use of antihypertensive agents and/or a systolic blood pressure of $>160 \mathrm{mmHg}$ and/or diastolic pressure of $>95 \mathrm{mmHg}$; 2) presence of diabetes, defined as the use of oral hypoglycemic agents or insulin, fasting blood glucose level of $\geq 120 \mathrm{mg} / \mathrm{dl}$ and/or hemoglobin A1C level of $\geq 5.9 \%$; 3) current smoking, defined as a history of smoking over the preceding three months; 4) presence of hypercholesterolemia, defined as the use of antihyperlipidemic agents or a serum cholesterol level $>220 \mathrm{mg} / \mathrm{dl}$.

For the evaluation of renal function, serum creatinine was examined on admission. Left ventricular fractional shortening (FS) was measured on an echocardiogram within 3 days of admission, and FS $\leq 30 \%$ was regarded as evidence of cardiac hypokinesis.

\section{Outcome parameters}

All patients of the two subject groups were evaluated with the following parameters: patient background characteristics including age, gender, and modified Rankin Scale (mRS) (13) before onset of symptoms, as determined by interviews with patients or family members. Patients were also evaluated by NIHSS on admission (NIHSS $)_{0}$ and at day 10 $\left(\mathrm{NIHSS}_{10}\right)$, and change in status during the acute phase was assessed as the difference between NIHSS $_{10}$ and NIHSS $_{0}$. Other clinical outcomes including drug -induced complications, length of hospital stay, and mRS at discharge were investigated. The mRS scores were evaluated by physicians, with $\mathrm{mRS} \leq 1$ designated as a "favorable outcome group," and $\mathrm{mRS} \leq 2$ as an "independent outcome group." In addition, the patients in these two subject groups were divided into four subgroups, according to clinical severity as assessed by NIHSS $_{0}$, as follows: quartile 1 (mild deficit group) with $\mathrm{NIHSS}_{0} \leq 7$; quartile 2 (moderate deficit group) with NIHSS $_{0} 8$ to 15 ; quartile 3 (severe deficit group) with NIHSS $_{0} 16$ to 20; quartile 4 (very severe deficit group) with $\mathrm{NIHSS}_{0} \geq 21$.

Six months after their admission, a return postcard with a questionnaire about $\mathrm{ADL}$ was sent to each patient. On the postcard 7 items were listed referring to ADL status, based on mRS. Patients or their family members selected the item with the closest match, and returned the postcard. From these data, the status of ADL at 6 months was evaluated in each patient.

\section{Statistical analysis}

To investigate the efficacy of edaravone, the clinical characteristics of the two main subject groups (ED and control groups) were compared according to patients' background characteristics (age, gender, pre-onset mRS, NIHSS ${ }_{0}$, serum creatinine, left ventricular FS, medication during acute phase 
Table 1. Characteristics and Outcome of Study Subjects

\begin{tabular}{|c|c|c|c|c|c|c|c|c|c|c|c|c|c|c|c|}
\hline & \multicolumn{3}{|c|}{ 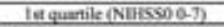 } & \multicolumn{3}{|c|}{ Ind quilike (NIISSS0 8-15) } & \multicolumn{3}{|c|}{ 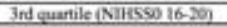 } & \multicolumn{3}{|c|}{ 4th qu rike (NIHSSO 21-40) } & \multicolumn{3}{|c|}{ Total } \\
\hline & $\begin{array}{c}\text { Earn } \\
\text { vone }\end{array}$ & Conenol & $p$ & $\begin{array}{l}\text { Edarn- } \\
\text { vone }\end{array}$ & Coetrol & p & $\begin{array}{l}\text { Edan } \\
\text { rone }\end{array}$ & Control & ? & $\begin{array}{c}\text { Idane } \\
\text { voos }\end{array}$ & Centrol & $p$ & $\begin{array}{l}\text { Edara: } \\
\text { vope }\end{array}$ & Contool & $p$ \\
\hline Number of patiets, a & 31 & 27 & & 34 & 27 & & 39 & 24 & & 37 & 23 & & 141 & 101 & \\
\hline $\begin{array}{l}\text { Ags. yo, mean } \\
\text { (median) }\end{array}$ & $14(74)$ & $74(76)$ & 0.517 & $78(90)$ & $73(74)$ & 0.038 & $s(m)$ & $79(x)$ & 0.328 & $81(80)$ & $79(80)$ & 0.474 & $n(78)$ & $76(7)$ & 0.375 \\
\hline Men $\%$ & 58 & 90 & 0.927 & 47 & 44 & $0 \times 30$ & 46 & 42 & ans & 4) & 4) & 0.986 & 45 & 48 & 0.914 \\
\hline $\begin{array}{l}\text { "miks before onser. } \\
\text { mean (modian) }\end{array}$ & $0.8(0)$ & $05(0)$ & 0.858 & aros & 120 & 0.062 & $13(0)$ & $0.6(0)$ & 0.142 & $1.7(0)$ & $1001)$ & Q.199 & $1.1(0)$ & $0 \times(0)$ & 0.553 \\
\hline Hypertension, $\%$ & 58 & as & 0.450 & 65 & 59 & 0.663 & 59 & 46 & 0.310 & 51 & 61 & 0.071 & ss & 53 & 0.512 \\
\hline Diabetes mellinis, $\$ 5$ & 19 & it & 0.481 & 24 & is & 0.395 & 21 & 17 & 0.786 & 24 & 9 & 0.178 & 22 & 13 & 0.091 \\
\hline $\begin{array}{l}\text { Hypercholesterolemia, } \\
5\end{array}$ & 19 & 19 & 0.935 & 9 & 22 & 0.167 & 3 & 13 & 0.150 & 8 & 17 & 0.412 & 9 & 18 & 0.053 \\
\hline Curmet noding, $\mathrm{S}$ & 13 & 15 & $>0.9 m$ & 21 & 19 & $>0.9 m$ & 10 & 17 & 0.467 & 22 & 17 & 0.752 & 16 & 17 & $>0.99$ \\
\hline $\begin{array}{l}\text { Senum cratinitici, } \\
\text { melit, mean (modtian) }\end{array}$ & $\begin{array}{l}0.8 \\
(0.8)\end{array}$ & $\begin{array}{l}0.8 \\
(0.8)\end{array}$ & 0.500 & $\begin{array}{l}1.1 \\
(0.75)\end{array}$ & $\begin{array}{l}0.8 \\
(0.8)\end{array}$ & 0312 & $\begin{array}{l}0.9 \\
(0.8)\end{array}$ & $\begin{array}{l}0.8 \\
(0.8)\end{array}$ & 0.653 & 100 & $\begin{array}{c}10 \\
(0.85)\end{array}$ & 0.104 & 0.9 & $\begin{array}{l}0.9 \\
(0.8)\end{array}$ & 0.738 \\
\hline $\begin{array}{l}\text { Fractional dortening } \\
\text { s } 305 \text {. } 5\end{array}$ & 26 & 22 & 0.750 & 20 & 22 & 0.465 & 13 & 13 & 0.970 & 35 & 35 & 0.978 & 22 & 23 & exss \\
\hline $\begin{array}{l}\text { "NIHSS, } \\
\text { (median) }\end{array}$ & $4(4)$ & $4(4)$ & 0.205 & (iil) & $12(12)$ & 0.578 & $18(18)$ & $18(18)$ & $0.7 \times 3$ & $26(31)$ & $30(31)$ & 0.137 & 169 & $15(14)$ & 0.328 \\
\hline Otapre wodum \% & 10 & 26 & 0.164 & 3 & 7 & $0.5 \mathrm{n}$ & 0 & 0 & & 0 & 0 & & 5 & 9 & 0.046 \\
\hline Mepurin \% & 44 & 48 & 0.792 & 29 & 49 & 0.17 & 39 & 38 & axys & 41 & 35 & $\operatorname{arcos}$ & is & 43 & 0.588 \\
\hline Argatrovan, $\%$ & 3 & 4 & $=0.99$ & is & 7 & 0.4 & 3 & 8 & 0.61 & 3 & 0 & $\times 9$ & 6 & 5 & 0.783 \\
\hline Glycend, $\mathrm{S}$ & 13 & 26 & 0.207 & 44 & 67 & $0.07 \%$ & 52 & 88 & 0.729 & 92 & 9 & $\times 090$ & 60 & 68 & 0.349 \\
\hline Aspirin, $\$$ & 10 & 22 & 0.270 & 24 & 0 & 0.007 & 13 & 4 & 0.394 & 11 & 4 & 0.641 & 14 & 8 & 0.197 \\
\hline Warfaris 4 & 61 & 78 & 0.176 & 59 & $\$ 0$ & 0000 & 59 & $n$ & 0.42 & 38 & 26 & 0.48 & 54 & 67 & 6046 \\
\hline $\begin{array}{l}\text { "NilHSS is meat } \\
\text { (medias) }\end{array}$ & $2(1)$ & 6(2) & 0.115 & $9(7.5)$ & $8(8)$ & 0.896 & $16(15)$ & $14(15)$ & 0.552 & $26(31)$ & $31(34)$ & 0.140 & $14(12)$ & $14(10)$ & 0.791 \\
\hline "NLHSS, - NIHSS, & $2(3)$ & $.2(1)$ & 0.013 & 3(3) & $4(3)$ & 0.936 & $2(3)$ & $4(4)$ & 0.452 & $0(0)$ & $4(0)$ & 0.351 & 2(3) & $1(2)$ & 0.385 \\
\hline Aoute remal finure, 5 & 0 & 0 & $>0.99$ & 3 & 4 & $>0.99$ & 3 & 0 & $=0.99$ & 0 & 4 & 0363 & 1 & 2 & $>0.99$ \\
\hline 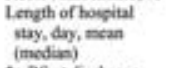 & 17(12) & $15(3)$ & 0.386 & $\begin{array}{c}18 \\
(155)\end{array}$ & 19 (1) & 0.025 & 1909 & $20(20)$ & 0.681 & 21 (15) & 22 (18) & 0.988 & $19(16)$ & $19(17)$ & 0.250 \\
\hline $\begin{array}{l}\text { "miRS at fischarge, } \\
\text { mean (median) }\end{array}$ & $2.1(2)$ & $2.1(1)$ & 0.766 & $3.7(4)$ & $3.7(4)$ & 0.081 & $47(5)$ & $4.5(5)$ & $0 \times 72$ & $5.0(5)$ & $5,4(5)$ & $\cos 2$ & $4.0(5)$ & $38(4)$ & 0.738 \\
\hline $\begin{array}{l}\text { (miks S1) at } \\
\text { discharge, } \$\end{array}$ & 39 & $\$ 6$ & 0.200 & 18 & It & 0.718 & 0 & 8 & 0.141 & 0 & 0 & $\times 090$ & 13 & 30 & $0.13 \mathrm{~s}$ \\
\hline 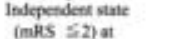 & $\pi$ & to & 0.400 & 21 & 19 & 08200 & 3 & 8 & 0.592 & 0 & 0 & $x$ & 21 & 26 & 0.417 \\
\hline discharges s & 3 & 7 & 089 & 0 & 4 & 0443 & 8 & 13 & 0 cost & 24 & 39 & 0224 & 9 & 15 & o1n \\
\hline
\end{tabular}

other than edaravone), status later during hospitalization or at discharge NIHSS $_{10}$, early improvement, drug-induced complications during admission, mRS, length of hospital stay), and mRS at 6 months after onset of symptoms. A similar statistical analysis was performed in each of the four subgroups segregated according to clinical severity as determined by NIHSS $_{0}$.

The clinical characteristics of the patients were compared between the two groups using the Mann Whitney U-test, $\chi^{2}$ test and Fisher's exact test. Statistical analysis was performed using a commercially available software package (StatView, version 5, SAS Institute Inc.,S Cary, NC USA). A value of $p<0.05$ was considered statistically significant.

\section{Results}

\section{Eligible patients}

A total of 340 patients with the diagnosis of cardioembolic stroke defined by TOAST criteria were admitted to our hospital within 24 hours after the onset of symptoms during the 24-month study period. A total of 101 patients were classified as control group; 141 as ED group; and 98 as excluded group.

\section{Clinical characteristics of subject groups}

Thrombolytic agents including intravenous tissue plasminogen activator (IV-tPA) or selective intra-arterial urokinase (IA-UK) were administered in 4 patients ( 2 of the ED group and 2 of the control group) in the hyperacute phase. One patient in the control group who was treated with IV-tPA had no recanalization during admission. The second patient in the control group was treated with IA-UK, which resulted in partial recanalization soon after treatment. Two patients in the ED group were treated with IA-UK, and partial recanalization was seen after the treatment in one but not in another.

\section{Stratified analysis of clinical characteristics of ED and control groups}

Table 1 shows the clinical characteristics among the patients of the ED and control groups in each subgroup stratified according to clinical severity. There were no differences in gender, NIHSS ${ }_{0}$, serum creatinine and cardiac hypokinesis, or in outcome parameters at discharge. In the moderate deficit group, patients were significantly younger and mRS before onset of symptoms was higher in the control group than in the ED group. Frequency of treatment during the acute phase with aspirin and warfarin was also significantly different in the moderate deficit group. Acute renal failure, which was thought possibly to be a drug-induced complication, was noted in 2 patients of each group during the acute phase, but the incidence did not differ significantly between the two groups. In the mild deficit group, early improvement was significantly greater in the ED group than in the control group (+2 versus $-2 ; p=0.013$ ).

\section{Discussion}

The present study was conducted to investigate the efficacy of edaravone in patients with cardioembolic stroke. Significant early improvement was noted in patients with mild deficits among the ED group, in comparison with the control group. Significant efficacy was not seen in the patients with moderate or severe deficits among the same 
Table 1. (Continued)

\begin{tabular}{|c|c|c|c|c|c|c|c|c|c|c|c|c|c|c|c|}
\hline & \multicolumn{3}{|c|}{ Istevartile (NIHSs 0-7) } & \multicolumn{3}{|c|}{ 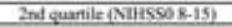 } & \multicolumn{3}{|c|}{ 3ed qumile (NIIHSo 16-20) } & \multicolumn{3}{|c|}{ 4h guartic (NIIHSSO 21-40\} } & \multicolumn{3}{|c|}{ Total } \\
\hline & $\begin{array}{l}\text { Edarn } \\
\text { vone }\end{array}$ & Contool & $p$ & $\begin{array}{l}\text { Idan: } \\
\text { venes }\end{array}$ & Conod & $p$ & $\begin{array}{l}\text { Itare: } \\
\text { vones }\end{array}$ & Conoud & $P$ & $\begin{array}{l}\text { Edara: } \\
\text { voese }\end{array}$ & Contod & $p$ & $\begin{array}{l}\text { Edara } \\
\text { wose }\end{array}$ & Coemend & $p$ \\
\hline $\begin{array}{l}\text { Niminer of followod } \\
\text { patiets } 6 \text { Mo afler } \\
\text { onset, a }\end{array}$ & 24 & 24 & & $\mathbf{m}$ & 20 & & 27 & 17 & & 28 & 19 & & 109 & $\$$ & \\
\hline $\begin{array}{l}\text { "miRS } 6 \mathrm{Mlo} \text { after } \\
\text { otoet, mean (modian) }\end{array}$ & $\begin{array}{l}1.6 \\
(0.5)\end{array}$ & $16(1)$ & 2.604 & $3.4(4.9)$ & $330)$ & a.8s & $43(5)$ & $3 x(5)$ & 0.690 & $53(5)$ & 5.t (6) & 0.559 & $3.7(5)$ & 330 & 0.476 \\
\hline $\begin{array}{l}\text { Favonble ouberne } \\
\text { (miles } 511) 6 \mathrm{Mo} \\
\text { ather onset, } \mathrm{S}\end{array}$ & 67 & 6? & $>0.90$ & 50 & 20 & 0.430 & 11 & 2 & 0.227 & 4 & 5 & $>0.90$ & 27 & 33 & 0.378 \\
\hline 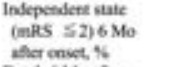 & 67 & 7 & 0.530 & 57 & 40 & 0.812 & 11 & 20 & a.227 & 4 & 5 & $>0.999$ & 28 & 41 & 0.006 \\
\hline 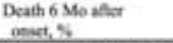 & 8 & 13 & $x=000$ & 3 & 15 & 0.289 & 19 & 2 & 0.473 & is & 53 & $0.6 \%$ & 19 & 26 & 0.254 \\
\hline
\end{tabular}

group. Patients with independent outcome status (mRS $\leq 2)$ were noted more frequently in the ED group than in the control group. Other clinical outcomes in the ED group were not significantly different from those in the control group.

To our knowledge no previous studies have reported that early improvement of neurological deficits is seen in acute stroke patients treated with edaravone. In patients with mild deficit among the control group, early worsening (mean NIHSS $_{10^{-}}$NIHSS $_{0}$ of -2) was found, thereby the effect of edaravone may retard the progression of ischemia, which may represent the natural course of the disease. Toyoda and colleagues (9) reported a historical-controlled study in which edaravone decreased brain edema and reduced fatalities in patients with unilateral internal carotid occlusion and severe deficits NIHSS $_{0}$ of 21). These investigators included in their series of patients any clinical category of ischemic stroke including cardioembolic stroke. In our study, however, early improvement was not seen in patients with severe or very severe deficits, although the study design was comparable to Toyoda's study. This discrepancy in the results of early improvement in patients with severe deficits between the two studies may be due to the differences in inclusion criteria and outcome parameters.

Efficacy in long-term outcomes with edaravone treatment in acute ischemic stroke has previously been observed in a study reported by the Edaravone Acute Brain Infarction Study Group (1). In this study, outcome parameters were not analyzed in subgroups stratified according to clinical severity, although enrolled patients were those with milder deficits (64\% of patients awake and $90 \%$ alert) than in our study. Also in this study, there was no limitation of the inclusion criteria on clinical categories, whereas in our study candidates were limited to those with cardioembolic stroke. In another historical-controlled study the efficacy of edaravone on short-term outcomes in patients with atherothrombotic stroke was reported (10). Also, it seems possible that the differences in heterogeneity or other features between the two studies are responsible for the observed discrepancies. To solve this problem, we propose that additional studies be undertaken to investigate the efficacy of edaravone in patients stratified according to stroke severity with methods of stratified design similar to our studies, or retrospective studies in which eligible patients are matched in neurologi- cal severity.

We hypothesize that there may be two pathophysiological reasons why edaravone was ineffective in our patients with severe deficits, as reported in the review of Gladstone and colleagues (14). First, the dose of $60 \mathrm{mg}$ per day of edaravone that we described may have been too small to scavenge free radicals in the large ischemic penumbra in patients with severe stroke deficits. Since dosages have often been based on preclinical studies of infarct size in species such as rats (2-7) rather than on behavioral studies in primates, this dose may be inadequate for the treatment of patients. Gladstone et al stated that preclinical studies rely on infarct size to judge therapeutic efficacy, whereas clinical trials rely on behavioral outcomes (14). Second, in the present study, some patients with severe deficits may have missed therapeutic windows at the time of treatment with edaravone. In many of the patients in the present study edaravone was administrated $>6$ hours after the onset of symptoms, at a time when thrombolysis had proved to be ineffective. Such patients with delayed administration of edaravone may not have experienced its full effects. Gladstone et al cited this as a concern in attempting to reconcile the results of negative stroke trials (14). Preclinical studies have often used very short time windows for drug administration, whereas clinical trials may allow longer time windows.

In the present study, the long-term efficacy of edaravone at 6 months was evaluated from values of mRS. However this value could be influenced by various other factors such as the occurrence of clinical complications and whether or not physical rehabilitation was employed. Gladstone et al stated that choice of outcome measures can determine the success of a clinical trial to a greater extent than actual drug efficacy (14). It is clearly necessary for studies in the future to investigate both early improvement and long-term outcomes, such as ADL and fatality rates.

The present study had several limitations. This was a historical-controlled and open label study but not a randomized controlled trial. Subgroup stratification resulted in smaller numbers for analysis. These considerations require caution in interpreting our results. Perhaps the bias in our study was reduced to some extent by the fact that there was no difference in $\mathrm{NIHSS}_{0}$ between the ED and control groups, although frequencies of treatment with aspirin and warfarin were significantly different in the moderate deficit 
group. In the present study we also investigated long-term outcomes by assessing mRS 6 months after the admission, based on return postcards sent to the patients or their family members, and this method may limit accuracy. To solve this problem, we should evaluate the functional outcome of patients through consultation or direct interview. Moreover, complications including metabolic syndrome, renal failure and heart failure may affect the clinical outcome in the patients with acute ischemic stroke. In our study, the severity of these complications was evaluated by past history, with only minor differences noted between the ED and control groups. It remains uncertain whether this type of analysis is sufficiently robust to serve as a reliable predictor of clinical outcomes.

In conclusion, based on our findings it is hypothesized that edaravone treatment may be effective in causing early neurological improvement in cardioembolic stroke patients with only mild neurological deficits. To further investigate this issue, it is recommended that double-blind, randomized placebo-controlled studies of edaravone treatment be carried out in subgroups stratified according to clinical severity.

\section{References}

1. The Edaravone Acute Brain Infarction Study Group. Effect of a novel free radical scavenger, edaravone (MCI-186), on acute brain infarction. Randomized, placebo-controlled, double-blind study at multicenters. Cerebrovascular Diseases 15: 222-229, 2003.

2. Abe K, Yuki S, Kogure K. Strong attenuation of ischemic and postischemic brain edema in rats by a novel free radical scavenger. Stroke 19: 480-485, 1988.

3. Nishi H, Watanabe T, Sakurai H, Yuki S, Ishibashi A. Effect of MCI-186 on brain edema in rats. Stroke 20: 1236-1240, 1989.

4. Watanabe T, Yuki S, Egawa M, Nishi H. Protective effects of MCI-186 on cerebral ischemia: Possible involvement of free radical scavenging and antioxidant actions. J Pharmacol Exper Ther 268: 1597-1604, 1994.

5. Kawai H, Nakai H, Suga M, Yuki S, Watanabe T, Saito KI. Effects of a novel free radical scavenger, MCl-186, on ischemic brain damage in the rat distal middle cerebral artery occlusion model. J Pharmacol Exp Ther 281: 921-927, 1997.

6. Mizuno A, Umemura K, Nakashima M. Inhibitory effect of MCI186, a free radical scavenger, on cerebral ischemia following rat middle cerebral artery occlusion. Gen Pharmacol 30: 575-578, 1998.

7. Yamamoto T, Yuki S, Watanabe T, Mitsuka M, Saito KI, Kogure K. Delayed neuronal death prevented by inhibition of increased hydroxyl radical formation in a transient cerebral ischemia. Brain Res 762: 240-242, 1997.
8. Minematsu K, Yamaguchi T, Omae T. 'Spectacular shrinking deficit': rapid recovery from a major hemispheric syndrome by migration of an embolus. Neurology 42: 157-162, 1992.

9. Toyoda K, Fujii K, Kamouchi M, et al. Free radical scavenger, edaravone, in stroke with internal carotid artery occlusion. J Neurol Sci 221: 11-17, 2004.

10. Takabatake Y, Uno E, Wakamatsu K, Yamazaki N, Hashimoto N, Tsuchiya Y. The clinical effect of combination therapy with edaravone and sodium ozagrel for acute cerebral infarction. No to Shinkei-Brain\&Nerve 55: 589-593, 2003 (In Japanese article, Abstract in English).

11. Muir KW, Weir CJ, Murray GD, Povey C, Lees KR. Comparison of neurological scales and scoring system for acute stroke prognosis. Stroke 27: 1817-1820, 1996.

12. Goldstein LB, Jones MR, Matchar DB, et al. Improving the reliability of stroke subgroup classification using the Trial of ORG 10172 in Acute Stroke Treatment (TOAST) criteria. Stroke 32: 1091-1097, 2001.

13. Sulter G, Steen C, De keyser J. Use of Barthel Index and Modified Rankin Scale in acute stroke trials. Stroke 30: 1538-1541, 1999.

14. Gladstone DJ, Black SE, Hakim AM. Toward wisdom from failure: Lessons from neuroprotective stroke trials and new therapeutic directions. Stroke 33: 2123-2136, 2002.

(C) 2006 The Japanese Society of Internal Medicine http://www.naika.or.jp/imindex.html 